\title{
PDA survey of medical residents: e-books before e-mail
}

\author{
Penny Logan and Seana Collins
}

\begin{abstract}
Introduction - Increasingly, database vendors are allowing downloads of their products to personal digital assistants (PDAs). The Hospital Library at Capital Health in Halifax, Nova Scotia, has the opportunity to provide PDA versions of resources to our users. The purpose of this survey is to find out the current environment of PDA use among the medical residents as a basis for developing library training and support for this technology. Question - Are medical residents using PDAs, and if yes, what type of PDA are they using, what experience do they have using them, how do they think they will use them in a clinical setting, and what products do they think they will find valuable in their practice? Methods - A Web-based survey was developed using PollDaddy software. A message was sent to all the medical residents on rotation at Capital Health on 12 November 2008; the survey closed 12 December 2008. Setting The survey was developed by the Health Sciences Library of Capital Health. Capital Health is an academic health centre in Nova Scotia, Canada. The medical residents are affiliated with Dalhousie University Medical School. Participants - The participants were medical residents currently working in Capital Health hospitals. There were 55 respondents. Results - The majority of respondents own a PDA and have more than 1 year of experience using the device. They use PDAs to look up drug information, for messaging, and to consult e-books. More than $90 \%$ of those surveyed use PDAs in their clinical practice. The Palm platform is used by $64 \%$ of respondents while the iPhone is used by $24 \%$. Conclusion - Medical residents are using PDAs with a preference for the Palm platform. They are used for clinical decision making, and 61 different sources were listed as currently used by the medical residents. Implications for the library are to provide training for the Palm and to concentrate on resource-specific training, rather than basic training on how to use a PDA. Limitations - There are approximately 300 medical residents on rotation at any one time. We received 55 responses. We realized, too late, that people could select only one item for question 7

("Which resources do you use?"). However, most respondents provided lists of titles of PDA resources for question 8.
\end{abstract}

\section{Summary of survey results}

Medical students are early-adopters of technology, and the use of personal digital assistants (PDAs), especially by medical residents, has been increasing [1]. Medical schools are encouraging students to use PDAs:

$70 \%$ of all U.S. Medical Schools are currently enrolled in the ePocrates student discount program which enables students to obtain $50 \%$ off a 1 yr. subscription to ePocrates Rx Pro [2].

PDAs fit in your coat pocket, go wherever you go, and can be personalized with the programs you like to use and information you frequently need [3].

Of the 57 who answered the question" "Do you own a PDA?", 51 respondents or $89 \%$ said "yes". Of the 51 medi- cal residents who own a PDA, $96 \%$ of them have owned their device for longer than 12 months.

Eighty-five percent of users owned a PDA before they started their residency, and $91 \%$ agreed that they will use the PDA in a clinical setting.

Sixty-four percent of users are using a Palm device. Considering the relatively high cost of the iPhone and its only recent availability in Canada, the authors were surprised to find that $24 \%$ of respondents used iPhones. We speculate that there will be more iPhone users as this technology increases in availability and prices decline.

We were interested to find that $6 \%$ used BlackBerry and $6 \%$ used "other" smart phones. Combined with the number of respondents using the iPhone, $36 \%$ of users had some kind of smart phone.

P. Logan. ${ }^{1}$ Health Sciences Library, Capital Health, 1796 Summer Street, Room 2212, Halifax, NS B3H 3A7, Canada (e-mail: penny.logan@cdha.nshealth.ca).

S. Collins. Health Sciences Library, Capital Health, 1276 South Park Street, Room 5107, Halifax, NS B3H 2Y9, Canada (e-mail: seana.collins@cdha.nshealth.ca).

${ }^{1}$ Corresponding author.

${ }^{2}$ Two of the respondents were library staff testing the survey. 
The Palm Pilot (Palm) platform is still the leader for the number of available medical software programs [4]. However, although Palm currently has the majority of downloadable databases, there are challengers to its prominence. Both the basic version of Epocrates, Epocrates Rx (the free drug software), and the RxPro (priced) version are now available for the BlackBerry (see http://www.epocrates.com/products/ rxpro/blackberry.html), as are several e-books including Current Consult Medicine (see http://www.usbmis.com/store/ home.php?cat=24). Epocrates Rx is available for the iPhone (http:// www.epocrates.com/gomobile/find-your-perfect-device.html ). Skyscape supports the iPhone (http://www.skyscape.com/ intro/iphoneintro.aspx) and several medical textbooks are now available for the iPhone (see http://www. unboundmedicine.com/store/iphone).

Some schools are supporting the iPod Touch as the PDA of choice for medical students: "The Ohio State University College of Medicine is the only college currently using the iPod Touch to give to all its students for educational purposes" [5].

PDAs were most often consulted for drug information and doses (49 users or $65.3 \%$ ). This may relate to the availability of Epocrates Rx as a free resource. We note that electronic text books were rated as being used more often than e-mail, contrary to our expectation that messaging features such as calendar, telephone, and e-mail would be cited as the mostused features.

The fact that drug information ranked highest, and electronic text books came in fifth place and ahead of e-mail strengthens the hypothesis that medical residents rely on PDA resources as a clinical tool (Table 1).

Respondents listed 61 different resources that they currently use on their PDAs. Products named more than once are shown in Table 2. Epocrates is the most used, followed by several versions of Lexi-Comp. These survey results show that drug information is the most-used PDA software, followed by medical calculators.

Future surveys should ask the respondents which software products they get for free and for which they pay. Full comments from those surveyed are shown under question 8 in Appendix A, but two quotes show that cost is a factor for this user group:

I used to have Lexi Comp, which was excellent as it contained a dictionary as well as a great drug program, but it's very expensive.

I would have more if they were less expensive.

The survey asked about three products that the library currently has the ability to support: Natural Standard (a complementary medicine database), Pocket Consult (via MD Consult), and ACP's Pier (via Stat! Ref).

The majority of medical residents $(85.7 \%)$ said they would use both Pocket Consult and ACP's Pier. Only about half that number, $45.8 \%$, said they would use Natural Standard. This is a new product for the Capital Health Library, so this may reflect a lack of familiarity with this product. Also, residency training focusses predominantly on the procedures of conventional medicine, so a different cohort-perhaps practicing family physicians who will have more patients using complementary preparations-may find greater uses for a complementary medicine product.
Table 1. PDA features most used.

\begin{tabular}{ll}
\hline Function & $\begin{array}{l}\text { Features most used } \\
\text { by respondents }(\%)\end{array}$ \\
\hline Drug database & $65.3(49)$ \\
Calendar & $52.1(48)$ \\
Contacts & $43.8(48)$ \\
Text/telephone & $40.4(47)$ \\
E-textbook & $31.9(47)$ \\
E-mail & $29.5(44)$ \\
\hline
\end{tabular}

Note: Values in parentheses are the number of respondents $(n=51)$.

Table 2. PDA products currently used.

\begin{tabular}{ll}
\hline Products named more than once & $\begin{array}{l}\text { No. of times product } \\
\text { by respondents }\end{array}$ \\
\hline Epocrates & 26 \\
Lexi-Comp/Drugs/Interact/Suite & 11 \\
Medical calculator & 10 \\
5-Minute Clinical Consult & 7 \\
PEPID & 7 \\
Harrison's & 6 \\
Stedman's Medical Dictionary & 6 \\
Washington Manual & 5 \\
DSM-IV & 3 \\
Taber's & 3 \\
Tarascon & 3 \\
UpToDate & 3 \\
PalmEKG & 2 \\
Skyscape applications & 2 \\
Toronto Notes & 2 \\
\hline
\end{tabular}

\section{Implications for library training}

(i) Medical residents will not need basic PDA training because they have owned a PDA for some time and will be accustomed to the features of their PDAs.

(ii) The library should emphasize training using Palm software to meet the needs of the largest numbers of users.

(iii) Smart phone technologies are the tools of choice for $36 \%$ of these medical residents. Future training should include smart phone technologies, and regular surveys should be delivered to see which type of technology is the preferred one.

Full survey results follow in Appendix A.

\section{Directions for further research}

More research needs to be conducted on evaluating skills levels before and after training in the use of PDA software products.

\section{Acknowledgement}

The authors wish to acknowledge the technical help of Verona Leslie, library technician, in the development and implementation of the survey. 


\section{References}

1. Garritty C, El Emam K. Who's Using PDAs? Estimates of PDA use by health care providers: a systematic review of surveys. J Med Internet Res. 2006;8(2):e7.

2. American Medical Student Association [homepage on the Internet]. Reston (Va.): American Medical Student Association [accessed 31 Dec 2008]. AMSA overview of handheld devises. Available from: http://www.amsa.org/resource/pda.cfm.

3. Hurt A. How PDAs are changing the clinic and classroom. New Physician. 2007 Sept [accessed 5 Jan 2009];56(6). Available from: http://www.amsa.org/tnp/articles/article.cfx $? \mathrm{id}=387$.
4. iPod Touch - a replacement for your PDA? The Palmdoc Chronicles. Palmdoc [accessed 18 Dec 2008]. Available from: http://palmdoc.net/?p=1672.

5. An iPod Touch for every medical student. The Palmdoc Chronicles. [posted 14 Dec 2008; accessed 5 Jan 2009] Available from: http://palmdoc.net/?p=2078.

Appendix A begins on the following page. 
Appendix A. Survey results

\section{Survey Title: PDA}

\begin{tabular}{|c|c|c|}
\hline \multicolumn{2}{|c|}{ Q.1 Do you own a PDA? } & \multirow[b]{2}{*}{ Count } \\
\hline \multicolumn{2}{|l|}{ Answer } & \\
\hline No & & $6(11 \%)$ \\
\hline \multirow[t]{3}{*}{ Yes } & & $51(89 \%)$ \\
\hline & People who answered question: & $57(100 \%)$ \\
\hline & People who skipped question: & $0(0 \%)$ \\
\hline
\end{tabular}

\begin{tabular}{|c|c|c|}
\hline \multicolumn{2}{|c|}{ Q.2 How long have you had your PDA? } & \multirow{3}{*}{$\begin{array}{c}\text { Count } \\
49(96 \%)\end{array}$} \\
\hline Answer & & \\
\hline$>12$ months & & \\
\hline 6 months & & $2(4 \%)$ \\
\hline \multirow[t]{3}{*}{12 months } & & $0(0 \%)$ \\
\hline & People who answered question: & $51(89.5 \%)$ \\
\hline & People who skipped question: & $\mathbf{6}(10.5 \%)$ \\
\hline
\end{tabular}

\begin{tabular}{|l|l|l|}
\hline Q.3 What type of PDA do you use? & \\
\hline Answer & & Count \\
\hline Palm & & $32(64 \%)$ \\
\hline Other smartphone & & $3(6 \%)$ \\
\hline Blackberry & & $3(6 \%)$ \\
\hline iPhone & & People who answered question: \\
\hline & People who skipped question: & 12 (24\%) \\
\hline
\end{tabular}

\begin{tabular}{|l|l|l|}
\hline Q.4 Did you own a PDA before commencing your residency? & \\
\hline Answer & & Count \\
\hline No & & $8(15 \%)$ \\
\hline Yes & & 47 (85\%) \\
\hline
\end{tabular}




\begin{tabular}{|l|r|c|}
\hline \multicolumn{2}{|l|}{ Q.4 Did you own a PDA before commencing your residency? } & \\
\hline N/A & & $0(0 \%)$ \\
\hline & People who answered question: & $55(96.5 \%)$ \\
\hline & People who skipped question: & $\mathbf{2}(3.5 \%)$ \\
\hline
\end{tabular}

\begin{tabular}{|l|r|c|}
\hline \multicolumn{2}{|l|}{ Q.5 Do you use OR intend to use your PDA in a clinical setting? } & \\
\hline Answer & Count \\
\hline Yes & & $50(91 \%)$ \\
\hline No & People who answered question: & $5(9 \%)$ \\
\hline & People who skipped question: & $\mathbf{2}(96.5 \%)$ \\
\hline
\end{tabular}

\begin{tabular}{|c|c|c|c|c|c|c|}
\hline \multicolumn{6}{|c|}{$\begin{array}{l}\text { Q.6 Please rank your use of the PDA where } 1 \text { is the most often used feature and } 5 \text { is the least often } \\
\text { used feature: }\end{array}$} & \multirow[b]{2}{*}{ Count } \\
\hline & 1 (most) & 2 & 3 & 4 & 5 (least) & \\
\hline As a calculator & $5(10.6 \%)$ & $\begin{array}{c}13 \\
(27.7 \%)\end{array}$ & $\begin{array}{c}11 \\
(23.4 \%)\end{array}$ & $\begin{array}{c}13 \\
(27.7 \%)\end{array}$ & $5(10.6 \%)$ & 47 \\
\hline As a medical calculator & $9(19.1 \%)$ & $\begin{array}{c}18 \\
(38.3 \%)\end{array}$ & $9(19.1 \%)$ & $9(19.1 \%)$ & $2(4.3 \%)$ & 47 \\
\hline Calendar & $\begin{array}{c}25 \\
(52.1 \%)\end{array}$ & $4(8.3 \%)$ & $9(18.8 \%)$ & $5(10.4 \%)$ & $5(10.4 \%)$ & 48 \\
\hline Track patient information (log) & $2(4.4 \%)$ & $3(6.7 \%)$ & $5(11.1 \%)$ & $6(13.3 \%)$ & $\begin{array}{c}29 \\
(64.4 \%)\end{array}$ & 45 \\
\hline Drug information and doses & $\begin{array}{c}32 \\
(65.3 \%)\end{array}$ & $8(16.3 \%)$ & $0(\%)$ & $4(8.2 \%)$ & $5(10.2 \%)$ & 49 \\
\hline Electronic text book & $\begin{array}{c}15 \\
(31.9 \%)\end{array}$ & $\begin{array}{c}13 \\
(27.7 \%)\end{array}$ & $6(12.8 \%)$ & $8(17 \%)$ & $5(10.6 \%)$ & 47 \\
\hline As procedure log & $4(9.3 \%)$ & $3(7 \%)$ & $6(14 \%)$ & $7(16.3 \%)$ & $\begin{array}{c}23 \\
(53.5 \%)\end{array}$ & 43 \\
\hline As MP3 player & $7(15.9 \%)$ & $3(6.8 \%)$ & $8(18.2 \%)$ & $4(9.1 \%)$ & $22(50 \%)$ & 44 \\
\hline Web searching & $7(15.6 \%)$ & $9(20 \%)$ & $4(8.9 \%)$ & $3(6.7 \%)$ & $\begin{array}{c}22 \\
(48.9 \%)\end{array}$ & 45 \\
\hline Text message, telephone & $\begin{array}{c}19 \\
(40.4 \%)\end{array}$ & $4(8.5 \%)$ & $2(4.3 \%)$ & $3(6.4 \%)$ & \begin{tabular}{c|c}
19 & 0 \\
$(40.4 \%)$
\end{tabular} & 47 \\
\hline Email & $\begin{array}{c}13 \\
(29.5 \%)\end{array}$ & $5(11.4 \%)$ & $3(6.8 \%)$ & $3(6.8 \%)$ & \begin{tabular}{c|}
20 \\
$(45.5 \%)$
\end{tabular} & 44 \\
\hline Other uses (entertainment/games) & $2(4.5 \%)$ & $6(13.6 \%)$ & $\begin{array}{c}13 \\
(29.5 \%)\end{array}$ & $1(2.3 \%)$ & $22(50 \%)$ & 44 \\
\hline Contacts & $\begin{array}{c}21 \\
(43.8 \%)\end{array}$ & $\begin{array}{c}11 \\
(22.9 \%)\end{array}$ & $7(14.6 \%)$ & $2(4.2 \%)$ & $7(14.6 \%)$ & 48 \\
\hline \multicolumn{6}{|c|}{ People who answered question: } & $52(91.2 \%)$ \\
\hline \multicolumn{6}{|c|}{ People who skipped question: } & $5(8.8 \%)$ \\
\hline
\end{tabular}




\begin{tabular}{|l|r|c|}
\hline \multicolumn{2}{|l|}{ Q.7 What resources are installed on your PDA? } & \multicolumn{1}{c|}{ Count } \\
\hline Answer & & $4(9 \%)$ \\
\hline 5 Minute Clinical Consult & & $4(9 \%)$ \\
\hline Medical Dictionary & & $20(43 \%)$ \\
\hline Epocrates & & $2(4 \%)$ \\
\hline Harrison's & People who answered question: & $16(35 \%)$ \\
\hline Other (please list below) & People who skipped question: & $11(80.7 \%)$ \\
\hline
\end{tabular}

\begin{tabular}{|c|c|}
\hline $\begin{array}{l}\text { Q.8 What other resources are installed on your PDA that are not listed above and what resource do } \\
\text { you use most often via your PDA? }\end{array}$ & \\
\hline People who answered question: & $36(63.2 \%)$ \\
\hline People who skipped question: & $21(36.8 \%)$ \\
\hline
\end{tabular}

Other: resources:

Pepid

5 Minute Clinical Consult (I need a drug reference!)

Tabers, Harrisons, medical calculator, Toronto Notes

lexidrugs and lexi suite (5 minute clinical consult, infectious dis, etc)

I used to have Lexi Comp, which was excellent as it contained a dictionary as well as a great drug program, but it's very expensive

PEPID, MedCalc, MedRules, Eponyms

medcalc, ABG, I would have more if they were less expensive

All of the above and much more.

Pepid, Emergency Medicine, Med Calc

Lexi, John hopkins Abx, Calculators

UpToDate, Lexi-Drugs, Sanford, 5 min ID, Critical Care Handbook, Vaccine Handbook

Taber's, Tarascon, PepID, CinciACS, CinciStroke, PalmEKG, others

Why does the previous question only allow one answer? Most people have multiple medical apps installed. I have epocrates, harrison's, stedman's dictionary, medmath, lexicomp

Skyscape applications

all of the above, also DSM-IV

tarascon 
harrisons, PEPID CRC and dictionary, trying to get uptodate (not working well on palm tx, though),tarascon, torontonotes

skyscape, epocrtes, med calculators, netter msk

Lexidrug, lexi interact. DrDrug, A-Z drugs, Washingtom manual

Lexi-comp

Anatomy interactive, Drug dosing guide,

Pepid

Stedmans, Tabers

med calc and lexi drugs

Netter's Anatomy Flashcards

Medical calculator, uptodate, toronto notes, tarascon

HB fracture, gamuts, stedmans dictionary, 5 min ortho/emerg/sports, washington manual, peds drugs, OCM, anaesthesia drugs -- use epocrates the most

Air Sharing - allows to view almost any document type on iTouch

Lexidrugs

Evidence Medicine calculator, Mobile Differential Diagnosis (MGH), The Washington Manual - Psychiatry Survival Guide

StatRef

I use Epocrates the most. I also have med calc and a series of oncology nomograms, diagnosaurus and an epononym program

Washington manual of surgery

Up to Date, Pepid

Epocrates, MedCalc, Wikipedia

epocrates, lexi drugs, dsm-iv, $5 \mathrm{mcc}$, ferri guide, washington m anual, med lab ref, palmekg, psychpharm, i use dsmiv most i think

\begin{tabular}{|c|c|c|c|}
\hline \multicolumn{3}{|c|}{ Q.9 Are you interested in having free access to the following Capital Health Library PDA resources? } & \multirow[b]{2}{*}{ Count } \\
\hline & Yes & No & \\
\hline $\begin{array}{l}\text { Natural Standard (free one year subscription) Collection of systematic reviews of alternative and } \\
\text { complementary medicine for clinicians; evidence-based, peer reviewed: Palm, PocketPC formats. }\end{array}$ & $\begin{array}{c}22 \\
(45.8 \%)\end{array}$ & $\begin{array}{c}26 \\
(54.2 \%)\end{array}$ & 48 \\
\hline $\begin{array}{l}\text { MD Consult / POCKETConsult (free unlimited timeframe subscription) Access to selected journals in } \\
\text { full-text, daily news updates, clinical updates, weekly drug updates, calculators and medical textbook }\end{array}$ & $\begin{array}{c}42 \\
(85.7 \%)\end{array}$ & $\begin{array}{c}7 \\
(14.3 \%)\end{array}$ & 49 \\
\hline $\begin{array}{l}\text { STAT!REF PDA:Pier (ACP Pier's) (free unlimited ti meframe subscription) Physician's Information and } \\
\text { Education Resource (PIER) evidence-based disease modules, provide quick access to authoritative an }\end{array}$ & $\begin{array}{c}42 \\
(85.7 \%)\end{array}$ & $\begin{array}{c}7 \\
(14.3 \%)\end{array}$ & 49 \\
\hline \multicolumn{3}{|c|}{ People who answered question: } & $50(87.7 \%)$ \\
\hline \multicolumn{3}{|c|}{ People who skipped question: } & $7(12.3 \%)$ \\
\hline
\end{tabular}

\begin{tabular}{|l|r|r|}
\hline Q.10 Please Identify Yourself: & \\
\hline Answer & & Count \\
\hline Resident & 51 (77\%) & \\
\hline
\end{tabular}




\begin{tabular}{|l|l|c|}
\hline \multicolumn{2}{|l|}{ Q.10 Please Identify Yourself: } & \\
\hline "other" & & $13(20 \%)$ \\
\hline Allied Health Professional & & $1(2 \%)$ \\
\hline Physician & & $1(2 \%)$ \\
\hline Clinical Clerk & & $0(0 \%)$ \\
\hline Nurse & People who answered question: & $0(0 \%)$ \\
\hline Pharmacist & People who skipped question: & $\mathbf{5 4}(94.7 \%)$ \\
\hline
\end{tabular}

